\title{
The Reconstruction of a Bicolored Domino Tiling from Two Projections
}

\author{
A. Frosini and G. Simi \\ Università degli Studi di Siena \\ Dipartimento di Matematica \\ Via del Capitano 15 \\ 53100 Siena, Italy \\ frosini, simi@unisi.it
}

\begin{abstract}
We challenge the problem of the reconstruction of a bicolored domino tiling of a rectangle from its horizontal and vertical projections. We give two $N P$-completeness results after having defined two non equivalent and very natural notions of projections on a generic bicolored domino tiling. The more general problem of the reconstruction of monochromatic domino tilings is still left open.
\end{abstract}

Keywords. Domino tiling, Reconstruction Problem, $N P$-completeness.

\section{Introduction}

The aim of Discrete Tomography is the reconstruction of a discrete finite set of points in the $d$-dimensional integer lattice $\mathbb{Z}^{d}$ using projections on lower dimensional subspaces. Important applications of Discrete Tomography are in image processing, in reconstructing structures from data get by an electronic microscopy, in data security and data compression (regarding the projections as an encoding process and reconstruction as a decoding process of a given object), and in computer-aided tomography.

Interesting results have been achieved in reconstructing planar sets using projections on one, two or more mono-dimensional subspaces. In [2], Ryser studies the problem of how to reconstruct a binary matrix (which models bidimensional sets) from its projections and finds a P-time algorithm for it. In [1] the authors extend the above result to matrices with a finite number of different entries (which models colored bidimensional sets) and find the NP-completeness when $n>3$ ( $n$-colors problem). These results become relevant when applied to the algorithms for the reconstruction of polyatomic crystal structures.

In this paper we use standard techniques of Discrete Tomography in order to reconstruct tilings of rectangular subsets of the plane with two colored dominoes. The horizontal and vertical projections of the tilings are a priori knowledge. In [5] the author shows that the reconstruction problem of a bicolored domino tiling is at least as hard as the reconstruction problem of a three entries matrix $(3-$ color s problem) and leaves as an open problem its computational complexity.

A. Braquelaire, J.-O. Lachaud, and A. Vialard (Eds.): DGCI 2002, LNCS 2301, pp. 136 144 2002. (C) Springer-Verlag Berlin Heidelberg 2002 
Furthermore in [6] a polynomial time algorithm which reconstructs a bicolored domino tiling from one projection is given.

The paper is organized as follows: in Section 2 we give some general definitions about bicolored domino tilings, in Section 3 we find the computational complexity of the reconstruction problem on such a class using two orthogonal projections. This problem arose in [5]. In the last section we consider and solve the same reconstruction problem using the different and non equivalent notion of horizontal and vertical projections introduced in [6].

\section{Definitions}

Let us consider an infinite squared surface $\mathcal{S}$ composed by cells and consider a two length horizontal or vertical bar called dominoes. A rectangular subset of $\mathcal{S}$ of dimension $m \times n$ has a domino tiling if it could be completely covered with non overlapping dominoes. Furthermore, a bicolored domino tiling, is a domino tiling which use two different kinds of dominoes: white and black ones. Columns are numbered from 1 to $n$, starting from the leftmost one, while rows are numbered from 1 to $m$, starting from the topmost one. A vertical domino covering two cells $c_{i, j}$ and $c_{i+1, j}$ is said to start on line $i$ and end on line $i+1$, while an horizontal domino covering two cells $c_{i^{\prime}, j^{\prime}}$ and $c_{i^{\prime}, j^{\prime}+1}$ is said to start on column $j^{\prime}$ and end on column $j^{\prime}+1$.

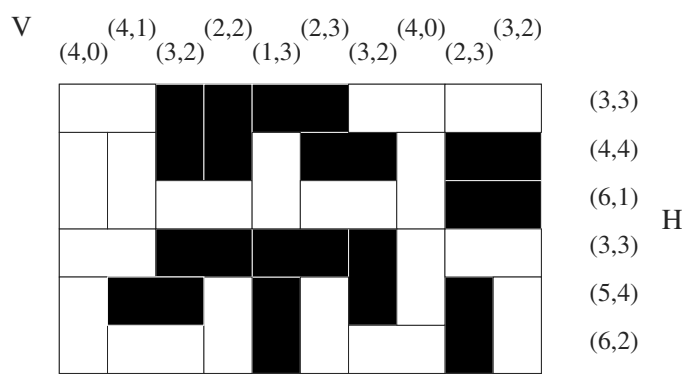

Fig. 1. A bicolored domino tiling of size $6 \times 10$ and its horizontal and vertical projections.

Let $B$ be a bicolored domino tiling of dimension $m \times n$, we define

$H=\left(\left(w_{1}, b_{1}\right), \ldots,\left(w_{m}, b_{m}\right)\right)$ the vector of the horizontal projections of $B$, where $w_{i}$ is the number of white dominoes which intersect at least one cell of line $i$ and $b_{i}$ is the number of black dominoes which intersect at least one cell of line $i$, for each $1 \leq i \leq m$.

$V=\left(\left(w_{1}^{\prime}, b_{1}^{\prime}\right), \ldots,\left(w_{n}^{\prime}, b_{n}^{\prime}\right)\right)$ the vector of the vertical projections of $B$, where $w_{j}^{\prime}$ is the number of white dominoes which intersect at last one cell of column $j$ and $b_{j}^{\prime}$ is the number of black dominoes which intersect at least one cell of column $j$, for each $1 \leq j \leq n$. 
The two vectors $H$ and $V$ do not univocally determine the number of horizontal (vertical as well) white dominoes on each row. The same holds for the black dominoes.

Through the paper we will indicate with $B$ a bicolored domino tiling of dimension $m \times n$; the vectors of horizontal and vertical projections of $B$ will be denoted with $H$ and $V$ in Section 3 and with $R$ and $C$ in Section 4 .

\section{The Reconstruction of a Bicolored Domino Tiling Consistent with Two Given Projections}

We define the two problems:

$\operatorname{ConsistenCY}_{(H, V)}($ b.d.t $)$.

Instance: two vectors $H \in(\mathbb{N} \times \mathbb{N})^{m}$ and $V \in(\mathbb{N} \times \mathbb{N})^{n}$.

Question: does there exist a bicolored domino tiling such that its horizontal and vertical projections are $H$ and $V$ respectively?

$\operatorname{ReCONSTRUCTION}_{(H, V)}($ b.d.t $)$.

Instance: two vectors $H \in(\mathbb{N} \times \mathbb{N})^{m}$ and $V \in(\mathbb{N} \times \mathbb{N})^{n}$.

Output: a bicolored domino tiling such that its horizontal and vertical projections are $H$ and $V$.

In $[5$ the author made a step towards the solution of the 3 -colors problem by reducing its instances to instances of $\operatorname{ConsISTENCY}_{(H, V)}$ (b.d.t.) so that if this problem allowed a $P$-time solution, then also 3 - colors would.

Unfortunately in this paragraph we prove that $\operatorname{CoNsisTENCY}_{(H, V)}($ b.d.t. $)$ is $N P$ complete: this prevent us from making any conjecture about the complexity of the 3 - colors problem. We achieve the above mentioned result by using a reduction which involves the $N P$-complete problem PARTITION (see [3]).

\section{PARTITION}

Instance: a finite sequence of integers $A=\left\langle a_{1}, \ldots, a_{k}\right\rangle$. Question: let $J=\{1, \ldots, k\}$. Is there $J^{\prime} \subset J$ such that

$$
\sum_{j \in J^{\prime}} a_{j}=\sum_{j \in J-J^{\prime}} a_{j} ?
$$

Lemma 1. Let $B$ be a bicolored domino tiling and $H$ its horizontal projection. The number of white dominoes in $B$ is lower bounded by $\frac{w_{1}+\cdots+w_{m}}{2}$ and upper bounded by $w_{1}+\cdots+w_{m}$.

Proof. We get the lower bound when in $B$ there are only vertical white dominoes so that each of them is counted in two different entries of $H$. We get the upper bound when in $B$ there are only horizontal white dominoes, so that each of them is counted only in one entry of $H$. 
Theorem 1. Consistency $_{(H, V)}$ (b.d.t.) is NP-complete.

Proof. The problem obviously belongs to $N P$.

Let $A=\left\langle a_{1}, \ldots, a_{k}\right\rangle$ be an instance $I$ of PARTITION with $\sum_{i=J} a_{i}=2 s$. We want to define in polynomial time $k-1$ instances $I_{1}^{\prime}, \ldots, I_{k-1}^{\prime}$ of the problem $\operatorname{ConsisTENCY}_{(H, V)}($ b.d.t.) such that a solution for $I$ exists if and only if a solution for at least one of the $I_{1}^{\prime}, \ldots, I_{k-1}^{\prime}$ exists. The index $i$ of the generic instance $I_{i}^{\prime}$ represents the cardinality (which is obviously less than, but not equal to $k$ ) of the set $J^{\prime}$.

Let us construct $I_{i}^{\prime}$ and let $B_{i}$ be one of its solutions, if it exists.

We define $H \in(\mathbb{N} \times \mathbb{N})^{4 s}$ and $V \in(\mathbb{N} \times \mathbb{N})^{3}$ as follows:

- $H$ is composed by couples which can be arranged in $k$ blocks, each of them encoding a different element of $A$. The height of the $j$-th block is $2 a_{j}$ :

$$
H=(\underbrace{(1,1),(2,1), \ldots,(2,1),(1,1)}_{\text {block } 1 \text { of length } 2 a_{1}}, \ldots, \underbrace{(1,1),(2,1), \ldots,(2,1),(1,1)}_{\text {block } k \text { of length } 2 a_{k}})
$$

$-V$ is composed by 3 couples:

$$
V=((s, s+i),(2 s-k, 2 k),(s, s+k-i))
$$

(see Figure 2 $a)$ ).

We make some useful remarks:

let us consider the first block of horizontal projections of $H$, and let $B_{i}^{1}$ be a bicolored domino tiling consistent with it as shown in Figure $2 b$ ) and $c$ ), it holds:

i) by Lemma 1, the minimum number of white dominoes in $B_{i}$ which are required by $H$ is $\sum_{j=1}^{k} 2 a_{j}-1=4 s-k$, which is also the maximum number of white dominoes required by $V$ (we compute it by adding $w_{1}^{\prime}+w_{2}^{\prime}+w_{3}^{\prime}$ of $V$, that is $s+(2 s-k)+s)$. It follows that $B_{i}$ has exactly $4 s-k$ white dominoes;

ii) again by Lemma 1 the minimum number of white dominoes in $B_{i}^{1}$ is

$$
\frac{1}{2}(\underbrace{1+2+\cdots+2+1}_{2 a_{1} \text { times }})=2 a_{1}-1
$$

Figure $2 c$ ) shows a solution having a higher number of white dominoes;

iii) since $B_{i}$ is composed by $k$ blocks, using $i$ ) we get that, for each $1 \leq j \leq k$, $B_{i}^{j}$ has exactly $2 a_{j}-1$ white dominoes, which are all vertical ones;

$i v$ ) from the previous remarks it follows that each $B_{i}^{j}$ has two horizontal black dominoes which are placed on the first and the last row of the block;

vi) since the number of black dominoes in the second column of $B_{i}$ is $2 k$ and remark iii) and $i v$ ) hold, we get that each $B_{i}^{j}$ has only two horizontal black dominoes in the first and on the last rows. 


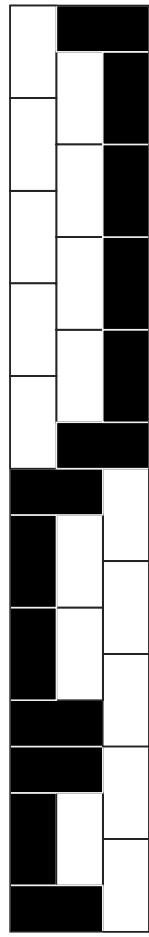

a)

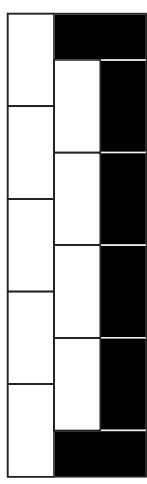

$(1,1)$

b)

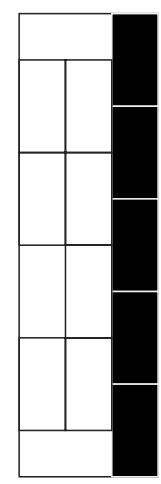

$(1,1)$

$(2,1)$

$(1,1)$

Fig. 2. The tiling which leads to a solution of the instance $A=\langle 5,3,2\rangle$ of PARTITION with $i=2$.

The above remarks imply that $B_{i}$ can assume only fixed configurations: each of them leads us to a solutions of PARTITION.

On line $1, B_{i}$ has a white vertical domino and a black horizontal domino; if the white domino is placed on column 1 , then the tiling of the whole first block $B_{i}^{1}$ is univocally fixed. We can consider such a tiling as the coding of the belonging or no-belonging of the index 1 to $J^{\prime}$ (see Figure $2 \mathrm{a}$ )). If the white domino is placed on column 3 we get a symmetrical tiling.

For the remaining $k-1$ blocks a similar behavior holds: by placing the first line of each of them we determine the whole tiling of the block.

On column $1, B_{i}$ has only the white dominoes belonging to the coding of $i$ elements of $A$ whose sum is $s$. The indexes of such elements forms the required set $J^{\prime}$.

On the other hand let $J^{\prime}$ be a solution for $I$ having cardinality $i$. We proceed by coding each element having index in $J^{\prime}$ by a block which has white dominoes 
in column 1 and each element having index in $J-J^{\prime}$ by a block which has white dominoes in column 3. The constructed tiling is one of the solutions of the instance $I_{i}^{\prime}$ of $\operatorname{ConsisTenCY}_{(H, V)}($ b.d.t.).

Corollary 1. The problem Reconstruction $(H, V)$ (b.d.t.) is NP-hard.

\section{A New Reconstruction Problem on the Class of Bicolored Domino Tilings}

In this section we give a different and very natural definition of projections for a bicolored domino tiling (as introduced in [6]). Through the section, the new vectors of horizontal and vertical projections will be referred to as $R$ and $C$ in order to distinguish them from $H$ and $V$ previously defined. In [6] the author shows a polynomial algorithm which solves RECONSTRUCTION $(R)$ (b.d.t.) and he leaves ReCOnstruction $(R, C)$ (b.d.t.) as an open problem. In spite of the two notions of projections are not equivalent, we prove the $N P$-completeness of $\operatorname{CONSISTENCY}_{(R, C)}($ b.d.t.) by reducing it to the problem PARTITION in a similar way as in the Theorem 1. This result solves a question proposed in 5 and [6]. However a general question about domino tilings remains still open: the computational complexity of the reconstruction of a domino tiling from two projections.

A new notion of projections.

Let $B$ be a $m \times n$ bicolored domino tiling, we define $R=\left(r_{1}, \ldots, r_{m}\right)$ and $C=$ $\left(c_{1}, \ldots, c_{n}\right)$ as the vectors of the horizontal and vertical projections respectively, where, for each $1 \leq i \leq m$ and $1 \leq j \leq n, r_{i}$ is the number of cells covered with a white domino on line $i$ and $c_{j}$ is the number of white cells covered with a white domino on column $j$.

Theorem 2. Let $H$ and $R$ be the two horizontal projections of $B$, there does not exist neither a function $f:(\mathbb{N} \times \mathbb{N})^{m} \rightarrow \mathbb{N}^{m}$ which maps $H$ into $R$ nor a function $g: \mathbb{N}^{m} \rightarrow(\mathbb{N} \times \mathbb{N})^{m}$ which maps $R$ into $H$ (this means that the two notion of projections are not equivalent).

Proof. examples $a$ ) and $b$ ) of Fig. 3 shows that a function $f$ which maps $H$ into $R$ can not be defined. Examples $c$ ) and $d$ ) prevent us from defining the function $g$ which maps $R$ into $C$.

The same result holds if we use the vertical projections $V$ and $C$.

Again a proof of NP-completeness which involves PARTITION.

Theorem 3. Consistency $(R, C)($ b.d.t) is NP-complete 


$\begin{array}{cc}H & R \\ (1,1) & 2 \\ (2,1) & 2 \\ (2,1) & 2 \\ (1,1) & 2\end{array}$

a)

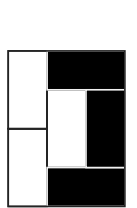

b) $\begin{array}{cc}H & R \\ (1,1) & 1 \\ (2,1) & 2 \\ (2,1) & 2 \\ (1,1) & 1\end{array}$

Fig. 3. Examples showing the non equivalence between $H$ and $R$.

Proof. The problem obviously belongs to $N P$. Let $A=\left\langle a_{1}, \ldots, a_{k}\right\rangle$ be an instance $I$ of PARTITION, $J=\{1, \ldots, k\}$ and $\sum_{i \in J} a_{i}=2 s$. We want to construct in polynomial time an instance $I^{\prime}$ of Consistency $_{(R, C)}($ b.d.t) such that a solution of $I$ exists if and only if a solution of $I^{\prime}$ exists.

We define $R \in \mathbb{N}^{4 s}$ and $C \in \mathbb{N}^{3}$ as follows:

- $R$ is composed of $k$ blocks such that, for each $1 \leq j \leq k$, the $j$-th block encodes the element $a_{j} \in A$ and its length is $2 a_{j}$ :

$$
R=(\underbrace{1,2, \ldots, 2,1}_{\text {block } 1 \text { of length } 2 a_{1}}, \ldots, \underbrace{1,2, \ldots, 2,1}_{\text {block } k \text { of length } 2 a_{k}}) ;
$$

$-C$ is the following vector:

$$
C=(2 s, 4 s-2 k, 2 s) .
$$

We prove that if $B$ exists, then it can assume only fixed configurations, each of them leading us to a solution of $I$.

We immediately note that, for each $1 \leq j \leq k$, the block $B^{j}$ of $B$ has $2 a_{j}-1$ white dominoes. The reconstructing procedure of $B$ is the following:

- on row 1 we place a vertical white domino on column 1 or 3 and an horizontal black domino covering the two remaining cells. No other tile is possible, otherwise we get stuck with the reconstruction on row 2. We choose to put the white domino on column 1 (a symmetrical behavior if we choose column 3 ) (see Figure $4 a)$ );

- on row 2 we place a vertical white domino and a vertical black domino in order to satisfy the second entry of $R$;

- on row 3 we have only a free cell where we place a vertical white domino;

- in an iterative manner we fill all the positions till row $2 a_{1}$ where the entry of $R$ is 1 and the cell on column 1 is covered with an ending vertical white domino. So the two free cells should be covered with one or two black dominoes;

- the above observations can be extended to each one of the $k$ blocks which has a black cell on column 2 on the first and the last row. The total number of black cells on such a column is $2 k$ and so the remaining $4 s-2 k$ cells are covered with white vertical dominoes, as requested from the second entry of $C($ see Figure $4 b))$; 


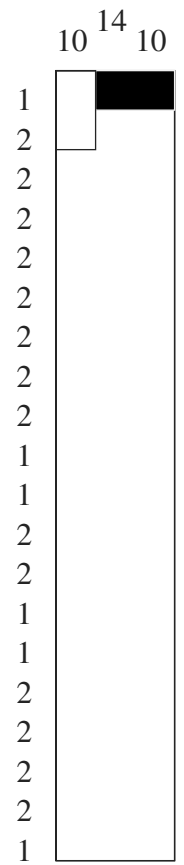

a)

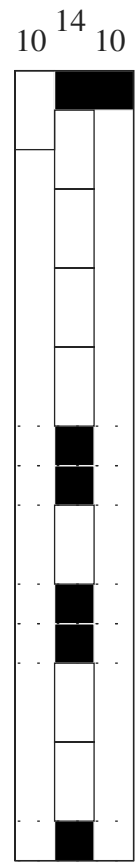

b)

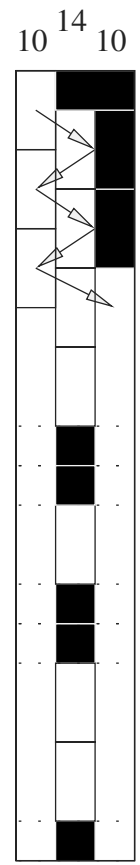

c)

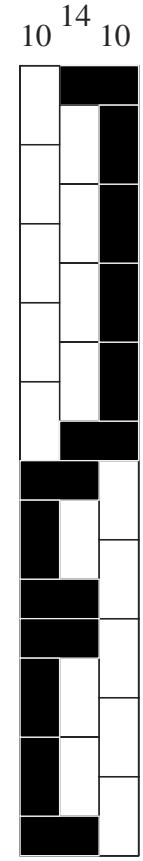

d)

Fig. 4. The reconstruction of a bicolored domino tiling associated to the instance $A=$ $\langle 5,2,3\rangle$ of PARTITION from $R$ and $C$.

- now we can tile column 1 and 3 of each block of $B$ with white or black vertical dominoes according to the entries of $C$ (see Figure 4 c) and $d)$ ).

Finally we observe that, for each $1 \leq j \leq k$, the block $j$ which codes the element $a_{j} \in A$ has the whole column 1 or the whole column 3 covered with $a_{j}$ vertical white dominoes. Furthermore in column 1 of $B$ we have $s$ vertical white dominoes which belongs to the blocks $j_{1} \ldots j_{t}$ and which code some elements $a_{j_{1}}, \ldots, a_{j_{t}}$ whose sum is $s$. The set $J^{\prime}=\left\{j_{1}, \ldots, j_{t}\right\}$ is the desired solution of the instance $I$.

On the other hand, if a solution of $I$ exists, it is easy to construct a solution for the corresponding instance $I^{\prime}$.

Corollary 2. The problem ReCOnstruction $(R, C)($ b.d.t.) is NP-hard.

\section{Conclusions}

In this paper we have studied and solved the computational complexity of the reconstruction problem on the class of the bicolored domino tilings using two different pairs of orthogonal projections. These two problems arose while searching 
a solution for the complexity of the 3 - colors problem and for the reconstruction of a monochromatic domino tiling from two projections. These two classical challenges are still left unsolved.

\section{References}

1. M.Chrobak, C.Durr, Reconstructing Polyatomic Structures from Discrete X-Rays: NP-Completeness Proof for Three Atoms, Theoretical Computer Science, 259:81-98, (2001),

2. H.Ryser, Combinatorial Mathematics, Mathematical Association of America and Quinn \& Boden, Rahway, New Jersey, (1963)

3. M.R.Garey, D.S. Johnson, Computers and intractability: A guide to the theory of NP-completeness, Freeman, New York, (1979)

4. C.Dürr, E.Goles, I.Rapaport, E.Rémila, Tiling with bars under tomographic constraints Theoretical Computer Science to appear.

5. C.Picouleau, Reconstruction of domino tiling from its two orthogonal projections Theoretical Computer Science, 255, pgg.437-447 (2001).

6. C.Picouleau, Reconstruction of a coloured domino tiling from its projections Theoretical Computer Science submitted.

7. G. J. Woeginger, The reconstruction of polyominoes from their orthogonal projections, Inf. Proc. Letters 77 pagg.225-229 (2001). 